\title{
Extraordinary elevation of the glass transition temperature of thin polymer films grafted to silicon oxide substrates
}

\author{
Ranjeet S. Tate, ${ }^{\text {a) }}$ David S. Fryer, ${ }^{\text {a) }}$ Silvia Pasqualini, Martha F. Montague, \\ Juan J. de Pablo, and Paul F. Nealey ${ }^{\text {b) }}$ \\ Center for NanoTechnology and Department of Chemical Engineering, University of Wisconsin, Madison, \\ Wisconsin 53706
}

(Received 9 November 2000; accepted 13 September 2001)

\begin{abstract}
We used local thermal analysis and ellipsometry to measure the glass transition temperatures $\left(T_{g}\right)$ of supported thin films of poly(4-hydroxystyrene) (PHS) and hydroxy terminated polystyrene (PS-OH). The films were spuncast from solution onto silicon oxide substrates and annealed under vacuum at elevated temperatures to graft the polymer to the substrate. Grafting was verified and characterized in terms of the thickness of and the advancing contact angle of water on the residual layer after solvent extraction. For PHS, each segment of the polymer chain was capable of grafting to the substrate. The thickness of the residual layer increased with increasing annealing temperature. For this polymer the critical thickness below which the $T_{g}$ of the film deviated from the bulk value was nearly $200 \mathrm{~nm}$ after annealing at the highest temperature $\left(190{ }^{\circ} \mathrm{C}\right)$; the $T_{g}$ of films $100 \mathrm{~nm}$ thick or less were elevated by more than $50{ }^{\circ} \mathrm{C}$ above the bulk value. For PS-OH films the polymer was only capable of grafting at one chain end, forming a brush layer at the substrate interface. The critical thicknesses for PS-OH films and the $T_{g}$ elevations were substantially higher than for ungrafted PS films, but were not as large as for PHS. The film thickness dependence of $T_{g}$ for PHS and PS-OH were well described as piecewise linear, consistent with a "dual-mechanism" model. (C) 2001 American Institute of Physics. [DOI: 10.1063/1.1415497]
\end{abstract}

\section{INTRODUCTION}

The material properties of amorphous polymeric structures exhibit size dependence when one or more of the dimensions of the systems are below $50-100 \mathrm{~nm}$. The dimension dependence of the glass transition temperature $\left(T_{g}\right)$ of free standing and supported ultra thin polymer films have been described extensively in the literature, ${ }^{1-15}$ and there are some reports of dimension dependent transport ${ }^{16-20}$ and mechanical properties ${ }^{21-24}$ in nanoscale polymeric systems as well. In most commercial applications of films and coatings, the thickness of the polymer does not approach the sub-100 $\mathrm{nm}$ scale and the phenomena referred to above do not affect the properties, processing, and usefulness of the materials. However, in the microelectronics industry, the largest section of the U.S. economy, dimension dependent properties of polymeric nanostructures are anticipated to pose significant challenges, particularly with respect to the sub- $100 \mathrm{~nm}$ patterning of photoresist by advanced lithography. ${ }^{25}$ To reach critical patterning dimensions of less than $100 \mathrm{~nm}$, for example, the industry may be forced to use ultra thin films of resist in conjunction with $157 \mathrm{~nm}$ and extreme ultraviolet $(13.4 \mathrm{~nm})$ lithography due to the opacity of organic materials at these wavelengths. In these systems, resist processing conditions may have to be optimized as a function of film thickness for control over the transport of small molecules such as photogenerated acids in films of chemically amplified resist, particularly during a post-exposure bake. The thermophysi-

\footnotetext{
${ }^{a}$ Both authors have contributed equally to this work.

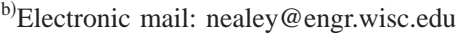

cal and mass transport properties of the films affect the sensitivity, resolution, contrast, and line edge roughness of the resist. Optimization may be difficult because diffusion coefficients of probe molecules change by orders of magnitude as the temperature is varied within $15^{\circ} \mathrm{C}$ above $T_{g}$.

Perhaps the most immediate challenge in lithography posed by dimension dependent properties of polymers, however, relates to the post-patterning mechanical stability of resist structures. As the widths and spacing of the polymeric nanostructures approaches $100 \mathrm{~nm}$, and aspect ratios are maintained at values of 3-4 for post-patterning processes such as reactive ion etching, the structures collapse during processing due to capillary forces. ${ }^{26}$ In the bulk, mechanical properties of amorphous polymers and their $T_{g}$ 's are related. ${ }^{27}$ The shear modulus, for example, increases substantially as the temperature of the sample is decreased below $T_{g} \cdot{ }^{28}$ It may be possible to actually improve the mechanical stability (at a constant temperature below $T_{g}$, i.e., room temperature) of nanoscopic polymer structures by designing systems in which the $T_{g}$ of the material increases as at least one of the dimensions of the system decreases.

In contrast to the $T_{g}$ of free-standing polymer films that decrease from the bulk value with decreasing thickness, others ${ }^{1}$ and $\mathrm{we}^{3}$ have observed that the $T_{g}$ of supported polymer films can decrease or increase depending on the substrate properties. By measuring the thermal expansion of thin polymer films with spectroscopic ellipsometry, Keddie, Jones, and Cory ${ }^{1}$ found that the $T_{g}$ of poly(methylmethacrylate) (PMMA) decreased from bulk $T_{g}$ on gold, but increased from the bulk $T_{g}$ on silicon oxide. Forrest et al. ${ }^{5}$ reported that the $T_{g}$ of thin films of poly(styrene) (PS) on silicon 
oxide decreased from the bulk value, but that $T_{g}$ of the supported films decreased to a lesser degree with decreasing film thickness than for free-standing films. Fryer et al. ${ }^{3}$ measured the $T_{g}$ of films of PS and PMMA on silicon oxide and silicon oxide treated with hexamethyldisilazane (HMDS). The $T_{g}$ of PMMA increased relative to the bulk value on silicon oxide (a polar surface) and decreased relative to the bulk value on silicon oxide treated with HMDS (a nonpolar and hence hydrophobic surface). The $T_{g}$ of PS decreased relative to the bulk value with decreasing film thickness on both types of substrates.

In a more recent study, Fryer et $_{\text {al. }}{ }^{29}$ showed that the effect of the substrate on the $T_{g}$ of thin films of PS and PMMA could be parameterized in terms of the interfacial energy between the polymer and the substrate. The interfacial energy between the substrates and either PMMA or PS was tuned by depositing self-assembled (SA) films of octadecyltrichlorosilane (OTS) on silicon oxide and exposing the SA films to increasing doses of soft x-rays in the presence of oxygen. During exposure, oxygen-containing groups (e.g., hydroxy, aldehyde, carboxy groups) are incorporated on the surface of the OTS. In this system, it is unlikely that the polymer penetrates into the SA film, or that the SA film reorganizes in response to different environments; the interfacial energy for PMMA and PS changes systematically with dose. Fryer et al. ${ }^{29}$ found that for sufficiently thin films: (i) the $T_{g}$ of PS and PMMA decreased relative to their respective bulk $T_{g}$ 's at low values of interfacial energy; (ii) the $T_{g}$ of PS and PMMA increased relative to their respective bulk $T_{g}$ 's at high values of interfacial energy; (iii) the deviation in $T_{g}$ from the bulk values of films of the same thickness of both PS and PMMA exhibited the same linear dependence on the interfacial energy for the range of interfacial energies and thickness investigated; and (iv) the deviation of $T_{g}$ from the bulk values increased with decreasing film thickness for constant interfacial energies.

Unfortunately, increases in the $T_{g}$ of polymer thin films associated with surface chemistry described above (different types of substrates or functionalized SA films) are not sufficient to induce a significant improvement in the mechanical properties of the materials at standard processing temperatures. Lithographically relevant film thicknesses for $157 \mathrm{~nm}$ or EUV lithography are approximately $100 \mathrm{~nm}$, and this is already considered an ultra thin resist in the industry. Increases in $T_{g}$ for polymer films in the literature referenced above do not occur for thicknesses greater than approximately $50 \mathrm{~nm}$, and to achieve a $30{ }^{\circ} \mathrm{C}$ increase in $T_{g}$ compared to the bulk value, at the highest value of interfacial energy in Ref. 29, the film thickness was $20 \mathrm{~nm}$. In this paper we demonstrate that the $T_{g}$ of a film $100 \mathrm{~nm}$ thick of a model resist resin, poly(4-hydroxystyrene) (PHS), is elevated above the bulk $T_{g}$ by as much as $55^{\circ} \mathrm{C}$ by grafting ${ }^{30}$ the polymer to the substrate. Grafted films of PHS $150 \mathrm{~nm}$ in thickness had $T_{g}$ 's approximately $30^{\circ} \mathrm{C}$ above the bulk value. We also show that the ability of every monomer in the PHS chain to bind to the substrate, and the consequent likelihood of multiple substrate attachment sites per chain, is responsible for the extraordinary behavior of the PHS films; the $T_{g}$ of hydroxy-terminated PS (PS-OH), which can only graft to the substrate at a chain end (and thus forms a brush layer near the substrate), increased above the bulk value only for film thickness less than $100 \mathrm{~nm}$, and then increased by only 15$20{ }^{\circ} \mathrm{C}$ for films $50 \mathrm{~nm}$ in thickness. The critical thickness (below which the $T_{g}$ of the film deviates from the bulk value) of the end-grafted PS-OH films is nearly double that of the ungrafted PS films $(\approx 50-60 \mathrm{~nm})^{8-11}$ but it is not as significant as the critical thickness of nearly $200 \mathrm{~nm}$ for the PHS films and the extraordinary $T_{g}$ elevation observed for PHS films as thick as $150 \mathrm{~nm}$.

Grafting, more so than the interfacial energy effects discussed above, has the unequivocal consequence of arresting chain mobility near the substrate. These results are discussed in terms of models of the $T_{g}$ behavior of thin films in which: (1) a mechanism is assumed to exist for mobility change as a function of temperature that is distinct from that responsible for bulk behavior and that dominates the bulk mechanism at low film thickness ${ }^{31,32}$ and (2) the mobility of the polymer chains is considered to be restricted in a layer at the substrate interface.

\section{EXPERIMENTAL SECTION}

\section{A. Materials}

Poly(4-hydroxystyrene) (PHS, $M_{w}=20.1 \mathrm{~kg} / \mathrm{mol}$ and $M_{w}=10.8 \mathrm{~kg} / \mathrm{mol}$, polydispersity index=2) was obtained from Hoechst Celanese (note that some suppliers refer to this polymer as poly(4-vinylphenol)). Commercial reagents, polystyrene $(\mathrm{PS}, 382 \mathrm{~kg} / \mathrm{mol})$, hexatriacontane $\left(\mathrm{C}_{36}\right)$, tetratetracontane $\left(\mathrm{C}_{44}\right)$, and hexacontane $\left(\mathrm{C}_{60}\right)$ were obtained from Aldrich (Milwaukee, WI). The chemicals were used without further purification, with the exception of tetrahydrofuran (THF) and styrene that were distilled prior to use in polymer synthesis. The silica gel used for flash chromatography was Merk Kiselgel 60 (230-400 mesh). Polished test grade silicon $\langle 100\rangle$ wafers were purchased from Tygh Silicon. Gel permeation chromatography was conducted on a Waters chromatograph with two styragel HT6E columns in series and a Waters 2410 differential refractometer with THF as the carrier solvent.

\section{B. Synthesis of hydroxy-terminated polystyrene}

The hydroxy-terminated initiator (1-hydroxy-2-phenyl-

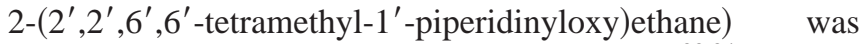
synthesized according to established procedure. ${ }^{33,34}$ The initiator was added to freshly distilled styrene along with a stir bar and sealed in a glass tube under vacuum. The living free radical polymerization reaction was performed at $125^{\circ} \mathrm{C}$ for $70 \mathrm{~h}$. The resulting (mono) hydroxy-terminated polystyrene (PS-OH) was analyzed by GPC and found to have $M_{W}$ $=100 \mathrm{~kg} / \mathrm{mol}$ and a polydispersity of 1.14 .

\section{Polymer deposition and grafting}

Prior to spin coating, silicon wafers were piranha cleaned, i.e., immersed in a solution of sulfuric acid and hydrogen peroxide (70:30 ratio by volume) for $30 \mathrm{~min}$ at $95^{\circ} \mathrm{C}$ and then rinsed with distilled water. PHS $(20.1 \mathrm{~kg} / \mathrm{mol}$ or $10.8 \mathrm{~kg} / \mathrm{mol}$ ) was dissolved in propylene glycol methyl 
ether acetate (PGMEA) to obtain solutions with concentrations ranging from $2 \%$ to $10 \%$ by weight. The solutions were filtered (Whatman Puradisc $0.2 \mu \mathrm{m}$ PTFE filters) and then spun cast (Solitec and Headway Research spinners) on the clean wafers at $1.8-2.5 \mathrm{krpm}$ to obtain films $70-350 \mathrm{~nm}$ thick. PS-OH was dissolved in toluene at concentrations between $1 \%$ and $3 \%$ by weight, filtered, and then spun cast to form films 42-216 nm thick. Samples were baked in a VWR Scientific vacuum oven at $130{ }^{\circ} \mathrm{C}, 160{ }^{\circ} \mathrm{C}$, and $190{ }^{\circ} \mathrm{C}$ for 3 $\mathrm{h}$ and allowed to cool to room temperature at approximately $1{ }^{\circ} \mathrm{C} / \mathrm{min}$. PS was dissolved in toluene at concentrations between $0.6 \%$ and $2.3 \%$ by weight, spun cast, annealed under vacuum at $130{ }^{\circ} \mathrm{C}$ for $3 \mathrm{~h}$, and cooled at $1{ }^{\circ} \mathrm{C} / \mathrm{min}$.

In order to characterize the grafting of the polymers to the substrates after annealing, the soluble portion of the film was dissolved from a piece of each wafer by placing it in hot solvent (toluene @ $90{ }^{\circ} \mathrm{C}$, PGMEA @ $110^{\circ} \mathrm{C}$ ) for $20 \mathrm{~min}$. The piece was then rinsed with acetone, blow-dried with nitrogen, rinsed with DI water, and again blow-dried with nitrogen before characterizing the residual polymer layer on the substrate.

Soxhlet extraction for selected samples was carried out in tetrahydrofuran (THF), a very strong solvent for PHS, at $40{ }^{\circ} \mathrm{C}$ for $6-8 \mathrm{~h}$. The samples were then rinsed and blowdried as above. The Soxhlet apparatus was purchased from ACE Glass.

\section{Characterization of polymer film and residual layer}

The thicknesses of the annealed polymer films as cast and those of the residual polymer layers were determined using a Rudolph Auto EL null ellipsometer. Measurements were made at three wavelengths: $632.8,546$, and $405 \mathrm{~nm}$, at an angle of incidence of $70^{\circ}$. The thickness was determined using FilmEllipse ${ }^{\circledR}$ software version 1.1 (Scientific Company Intl.) from measurements of the ellipsometric angles $\Psi$ and $\Delta$ made at all three wavelengths. The advancing contact angle of DI water on the residual layer was measured with a DataPhysics OCA20 goniometer using the sessile drop method and the associated SCA20 software.

\section{E. Glass transition temperature measurements}

We measured the $T_{g}$ 's of the films via local thermal analysis and ellipsometry. Local thermal analysis ${ }^{35,36} \mathrm{em}-$ ploys a microscopic thermistor element or a thermal probe that is mounted on a scanning probe microscope head. ${ }^{37}$ The method we follow is described in detail in a previous publication. ${ }^{3}$ Here we summarize.

During an experiment, the probe is placed in contact with the sample. The area of contact of the probe with the sample is approximately $1 \mu \mathrm{m}$ by $0.25 \mu \mathrm{m}$. Then the resistance (proportional to the temperature) of the probe is increased in steps and held constant at each step. The heat flux through the film at each set point resistance (temperature) is determined by measuring the power supplied to the probe in order to maintain the set point and subtracting from this the power supplied to the probe to maintain the same resistance when it is in air. At resistances corresponding to thermal events such as glass or melting transitions there is a change in the derivative of the differential probe power with respect to resistance. The most reproducible indicator of a thermal event was the onset of the change in slope and was best estimated by plotting the derivative of the power with respect to resistance. The temperature of the probe was calibrated to its resistance by a linear fit of the measured onset melting resistances to the known (via DSC) melting temperatures of long chain alkanes: hexatriacontane $\left(\mathrm{C}_{36}\right)$, tetratetracontane $\left(\mathrm{C}_{44}\right)$, and hexacosane $\left(\mathrm{C}_{60}\right)$. The thermal probes were obtained from Thermomicroscopes and mounted on a Topometrix Voyager SPM.

We followed a standard procedure for measuring the $T_{g}$ of thin films via null ellipsometry. ${ }^{1,11,38}$ The method consists of identifying the temperature at which the thermal coefficient of linear expansion of the thin film undergoes a change.

In Refs. 3 and 29 the authors established that the values of the glass transition temperature obtained via local thermal analysis were in good agreement with those obtained via ellipsometry for the same samples. In addition, $T_{g}$ values for PS films on $\mathrm{SiO}_{x}$ substrates measured via local thermal analysis (data from this work and from Ref. 3) agree well with values of $T_{g}$ measured by a large number of other techniques, most of these based on volumetric effects. The experimental consensus of the techniques is discussed recently in Ref. 20. References 8 and 9 contain extensive reviews and discussions of $T_{g}$ data for PS films on $\mathrm{SiO}_{x}$.

The glass transition temperature of the bulk polymer was measured with a Netzsch differential scanning calorimeter (DSC). Heating ramp rates used for measurement in the DSC were set to match the average ramp rates used with local thermal analysis (for comparison purposes) and varied from $3{ }^{\circ} \mathrm{C} / \mathrm{min}$ to $5{ }^{\circ} \mathrm{C} / \mathrm{min}$.

\section{RESULTS}

\section{A. Characterization of grafting of polymers to substrates}

PHS and PS-OH graft to the SiOH substrate in different ways, as shown in Fig. 1, in which we show only the grafted chains for the purpose of clarity. PHS, which has a hydroxy group on every monomer, can graft to the substrate at multiple points along the chain [Fig. 1(a)]. We refer to this as side grafting. On the other hand, $\mathrm{PS}-\mathrm{OH}$ can only graft to the substrate at the chain end, as shown in Fig. 1(b), and we refer to this as end grafting.

We measured the residual thickness $\left(h_{r}\right)$ and the advancing contact angle of $\mathrm{H}_{2} \mathrm{O}(\theta)$ of the polymers layers after solvent washes for films annealed for different times and temperatures. The values of the thickness and water contact angle for residual layers of PHS and PS-OH on silicon oxide substrates did not change (data not shown) after the first hour of annealing for all annealing temperatures studied. Neither $h_{r}$ nor $\theta$ changed appreciably as a function of the original thickness of the cast film. (For much thinner films, of thickness $<40 \mathrm{~nm}$, Jones et al. ${ }^{39}$ find that the thickness of the residual layer for end-grafted PS does depend on the film thickness as cast.) The thickness and contact angle of the residual layer did depend on the polymer (PHS or PS-OH), the molecular weight and the annealing temperature, as re- 


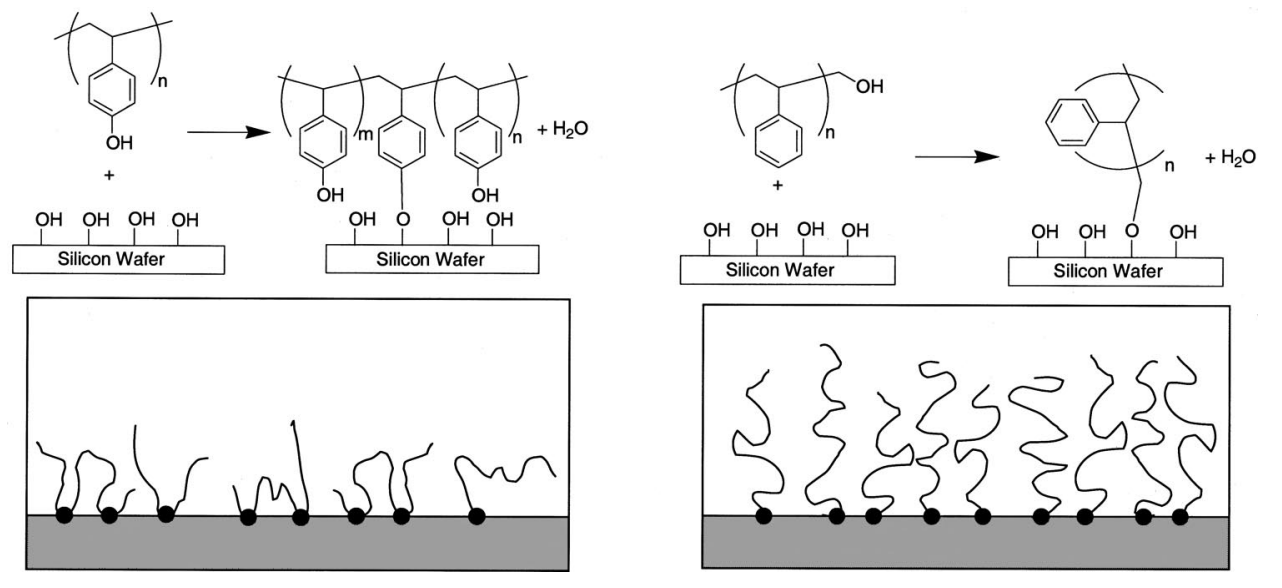

FIG. 1. Chemistry and schematic of grafting to a silicon oxide substrate of films of (a) side-grafted poly(4hydroxystyrene) and (b) end-grafted hydroxy terminated polystyrene. ported in Table I. We confirmed the uniformity of the residual layers by making measurements of both thickness and water contact angle at multiple spots on each sample.

The values of the contact angles on the residual layers indicate the presence of PHS films on the $\mathrm{SiO}_{x}$ substrates. After removing the soluble fractions of the PHS films as described in the Experimental Section, the contact angles of the residual films $\left(70-76^{\circ}\right)$ were between those of thick PHS films annealed above $T_{g}$ and rinsed in DI water $\left(79 \pm 1^{\circ}\right)$ and residual layers after Soxhlet extraction $\left(65 \pm 2^{\circ}\right)$. For comparison, the contact angle on annealed but otherwise untreated PHS films is $84 \pm 3^{\circ}$. We do not expect the contact angles for the films treated in different manners to be the same since rinsing in different solvents is known to cause rearrangement of the molecules at the surface and thus change the chemistry of the material presented at the surface. [See for example Sidorenko et al. ${ }^{40}$ who report switching the surface chemistry of poly(vinylpyridine) brushes with solvent treatment.]

We carried out a Soxhlet extraction of the soluble polymer layer from pieces cleaved from selected annealed samples. The values of $h_{r}$ for the residual layers after Soxhlet extraction (data not shown) were essentially the same as after the wash in PGMEA. This demonstrates that the standard solvent washes removed all the ungrafted polymer from the residual layer and that the polymer in the residual layer is truly grafted as opposed to just hydrogen bonded to the surface.

For PHS of $11 \mathrm{~kg} / \mathrm{mol}$ and $20 \mathrm{~kg} / \mathrm{mol}$, the residual layer thickness $h_{r}$ increased with increasing annealing temperature. Our interpretation of these results is that the extent of grafting increased with increasing temperature, however, no

TABLE I. Thickness $\left(h_{r}\right)$ and water contact angle $(\theta)$ for residual layers of PHS11, PHS20, and PS-OH annealed for $3 \mathrm{~h}$ at the temperatures shown. The radii of gyration of the polymers $\left(R_{g}\right)$ are included for comparison.

\begin{tabular}{lccccc}
\hline \hline Material & PHS11 & PHS11 & PHS20 & PHS20 & PS-OH \\
\hline$T_{\text {anneal }}\left({ }^{\circ} \mathrm{C}\right)$ & 130 & 190 & 160 & 190 & 130 \\
$R_{g}(\mathrm{~nm})$ & $5.4^{\mathrm{a}}$ & $5.4^{\mathrm{a}}$ & $7.6^{\mathrm{a}}$ & $7.6^{\mathrm{a}}$ & $9.4^{\mathrm{b}}$ \\
$h_{r}(\mathrm{~nm})$ & 0.9 & 2 & $3.9 \pm 0.1$ & $5.2 \pm 0.3$ & 19 \\
$\theta$ & $72 \pm 3^{\circ}$ & $76^{\circ}$ & $73 \pm 0.2^{\circ}$ & $75 \pm 2^{\circ}$ & $94 \pm 3^{\circ}$ \\
\hline \hline
\end{tabular}

${ }^{\mathrm{a}}$ Reference 41.

${ }^{\mathrm{b}}$ References 43 and 44 . information is available about the grafting density of the polymer. It is possible that more chains are covalently bound to the surface at higher annealing temperatures, and that more attachment sites are formed per chain. Note that if annealing at higher temperatures were to increase only the number of binding sites per chain, without increasing the number of bound chains, the residual layer thickness would be expected to decrease rather than increase.

To place the values of $h_{r}$ in context, we compare the residual film thicknesses to the radius of gyration $\left(R_{g}\right)$ of $\mathrm{PHS}^{41}$ shown in Table I. For PHS films annealed above the bulk $T_{g}$ of PHS $\left(148{ }^{\circ} \mathrm{C}\right), h_{r}$ is comparable to the estimated $R_{g}$ in Table I. For PHS11 films annealed at $130{ }^{\circ} \mathrm{C}$, below the bulk $T_{g}$ of PHS, $h_{r}$ is substantially less than the estimated $R_{g}$.

In the case of PS-OH, the advancing contact angle of $\mathrm{H}_{2} \mathrm{O}$ on the residual layers was $94 \pm 3^{\circ}$, consistent with the value $\left(92 \pm 2^{\circ}\right)$ reported for pure PS. ${ }^{42}$ The residual layer thickness $(19 \mathrm{~nm})$ was larger than the radius of gyration $\left(R_{g}\right)$ $(9.4 \mathrm{~nm})$ of bulk PS, ${ }^{43,44}$ indicating a stretching by a factor of about 2. These results are consistent with those of Mansky et $a l .{ }^{45}$ They reported a brush thickness of approximately 5 $\mathrm{nm}$ (twice the radius of gyration) obtained by annealing PS-OH $\left(M_{W} \approx 10 \mathrm{~kg} / \mathrm{mol}\right)$ films at $140{ }^{\circ} \mathrm{C}$ for 2 days and extracting the ungrafted polymer with solvent.

\section{B. Glass transition temperatures of PHS films}

We measured the glass transition temperatures of PHS films using local thermal analysis and null ellipsometry. $T_{g}$ (relative to the bulk value) of the films is plotted as a function of the reciprocal of the thickness in Fig. 2. Films of PHS20 annealed at $160{ }^{\circ} \mathrm{C}$ were measured using ellipsometry. $T_{g}$ 's of other films were measured primarily using local thermal analysis with a few samples also analyzed using ellipsometry in order to compare the results of the two techniques. We found good agreement between the two techniques, as had been established previously.,29 The $T_{g}$ measured for bulk samples of PHS with DSC, both 10.8 and $20.1 \mathrm{~kg} / \mathrm{mol}$, was $148^{\circ} \mathrm{C}$. For thick films of PHS (greater than $200 \mathrm{~nm}$ thick) the $T_{g}$ measured using local thermal analysis $\left(149 \pm 3{ }^{\circ} \mathrm{C}\right)$ and ellipsometry $\left(149 \pm 1{ }^{\circ} \mathrm{C}\right)$ corresponded to the bulk value. 


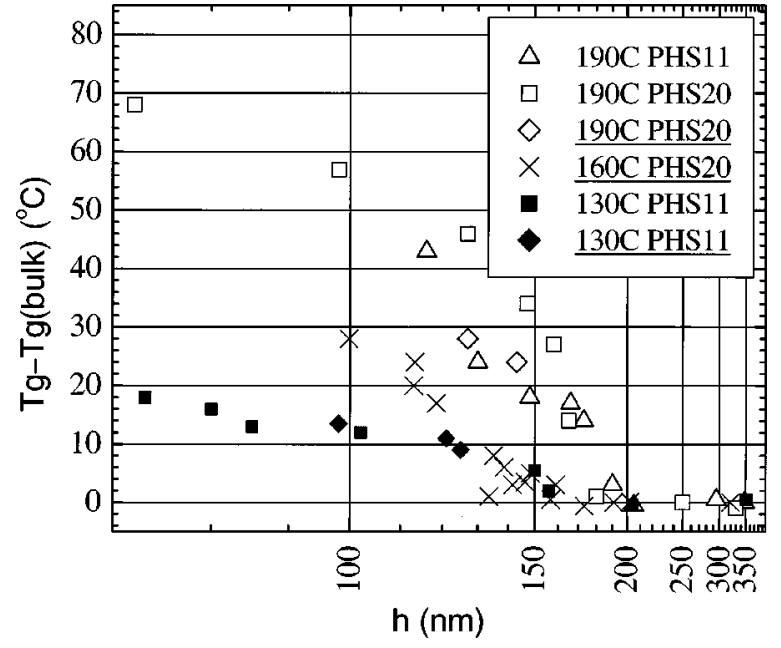

FIG. 2. Plot of the glass transition temperatures of PHS films grafted to silicon oxide, as a function of film thickness. Thickness is plotted on a reciprocal scale. Data are for films of two different molecular weights (as indicated in the legend) and annealed for $3 \mathrm{~h}$ at $130{ }^{\circ} \mathrm{C}$ (filled symbols), $160{ }^{\circ} \mathrm{C}(\times)$, and $190^{\circ} \mathrm{C}$ (open symbols). Underlined symbol legends refer to ellipsometry data; all other data were obtained using local thermal analysis.

In order to discuss the dependence of the glass transition temperature on the thickness and annealing temperature of PHS films, we introduce two parameters. The first parameter is the critical film thickness, $h_{c}$, below which the $T_{g}$ of the film deviates from the bulk $T_{g}$. For thicknesses less than $h_{c}$, $T_{g}$ increased with decreasing film thickness, and the data could be adequately fit to a line. We define the second parameter $\tau$, the thickness coefficient of the glass transition temperature, to be the (negative) slope of this line. Both these parameters can be obtained by fitting the $T g(h)$ data to the integral of a hyperbolic tangent function:

$$
T_{g}(h)=T_{g}^{\text {bulk }}+\frac{\tau}{2}\left(\Delta \operatorname{Ln}\left(2 \cosh \left(\frac{h-h_{c}}{\Delta}\right)\right)-h+h_{c}\right),
$$

where $\Delta$ is a parameter that allows for a finite width of the transition.

Recall from the previous subsection that the extent of grafting of PHS, as interpreted from the thickness of the residual layer $h_{r}$, is strongly correlated to the annealing temperature. We find that the thickness dependence of the glass transition temperature of PHS films varies with the extent of grafting in two ways, summarized in Table II.

First, the greater the extent of grafting, the greater the critical thickness at which deviations of $T_{g}$ from the bulk value are observed. For PHS11, $h_{c}$ increased from 179 \pm 9 $\mathrm{nm}$ at an annealing temperature of $130{ }^{\circ} \mathrm{C}$ to $198 \pm 12 \mathrm{~nm}$ at

TABLE II. Critical thickness $\left(h_{c}\right)$ and thickness coefficient of $T_{g}(\tau)$ of grafted films of PHS11, PHS20, and PH-OH annealed for $3 \mathrm{~h}$ at the temperatures shown.

\begin{tabular}{lccccc}
\hline \hline Material & PHS11 & PHS11 & PHS20 & PHS20 & PSOH \\
\hline$T_{\text {anneal }}\left({ }^{\circ} \mathrm{C}\right)$ & 130 & 190 & 160 & 190 & 130 \\
$h_{c}(\mathrm{~nm})$ & $179 \pm 9$ & $198 \pm 12$ & $147 \pm 15$ & $193 \pm 12$ & $110 \pm 11$ \\
$\tau\left({ }^{\circ} \mathrm{C} / \mathrm{nm}\right)$ & $0.16 \pm 0.02$ & $0.45 \pm 0.08$ & $0.5 \pm 0.1$ & $0.59 \pm 0.07$ & $0.32 \pm 0.7$ \\
\hline \hline
\end{tabular}

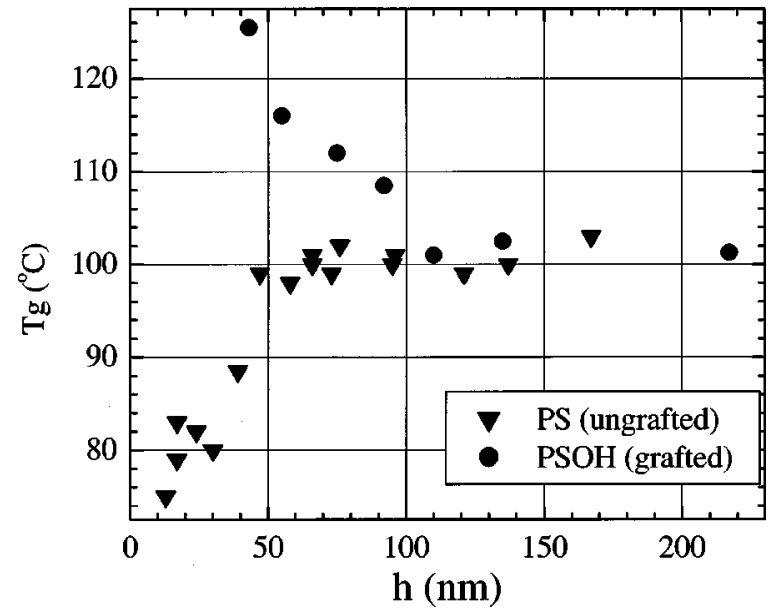

FIG. 3. Plot of the glass transition temperatures of PS-OH and PS films on silicon oxide (annealed at $130^{\circ} \mathrm{C}$ ), as a function of film thickness. All data were obtained using local thermal analysis.

an annealing temperature of $190{ }^{\circ} \mathrm{C}$. For PHS20 the effect of annealing temperature on $T_{g}$ is even more dramatic: $h_{c}$ increased from $147 \pm 15 \mathrm{~nm}$ at $160{ }^{\circ} \mathrm{C}$ to $193 \pm 12 \mathrm{~nm}$ at $190{ }^{\circ} \mathrm{C}$.

Second, for films of sub-critical thickness, the greater the extent of grafting the greater the increase in $T_{g}$ with decreasing film thickness. Specifically, for PHS11, $\tau$ changed from $0.16 \pm 0.016{ }^{\circ} \mathrm{C} / \mathrm{nm}$ at $130{ }^{\circ} \mathrm{C}$ (corresponding to $18{ }^{\circ} \mathrm{C} T_{g}$ elevation above the bulk value for a $72 \mathrm{~nm}$ thick film) to $0.45 \pm 0.08{ }^{\circ} \mathrm{C} / \mathrm{nm}$ at $190{ }^{\circ} \mathrm{C}$ (corresponding to $42{ }^{\circ} \mathrm{C} T_{g}$ elevation for a $117 \mathrm{~nm}$ thick film). Similarly, for PHS20, $\tau$ changed from $0.5 \pm 0.1{ }^{\circ} \mathrm{C} / \mathrm{nm}$ at $160{ }^{\circ} \mathrm{C}$ (corresponding to $26{ }^{\circ} \mathrm{C} T_{g}$ elevation for a $89 \mathrm{~nm}$ thick film) to $0.59 \pm 0.07{ }^{\circ} \mathrm{C} /$ $\mathrm{nm}$ at $190^{\circ} \mathrm{C}$ (corresponding to $68^{\circ} \mathrm{C} T_{g}$ elevation for a 72 nm thick film).

Finally, we found no substantial difference for the thickness dependent $T_{g}$ 's of PHS11 and PHS20 when annealed at the same temperature, hence there was no significant molecular weight dependence of $T_{g}(h)$, as expected for this range of molecular weights.

\section{Glass transition temperatures of PS-OH and PS films}

The $T_{g}$ for bulk samples of PS-OH measured via DSC was $102{ }^{\circ} \mathrm{C}$. For films (annealed at $130{ }^{\circ} \mathrm{C}$ ) more than 100 $\mathrm{nm}$ thick the $T_{g}$ measured using local thermal analysis corresponded to the bulk value $\left(101 \pm 3{ }^{\circ} \mathrm{C}\right)$. The critical thickness $h_{c}$ is $110 \pm 11 \mathrm{~nm}$ (see Fig. 3) below which the measured $T_{g}$ of the films increased relative to the bulk value at $\tau=0.33$ $\pm 0.07^{\circ} \mathrm{C} / \mathrm{nm}$, corresponding to a $T_{g}$ elevation of $25^{\circ} \mathrm{C}$ for a $43 \mathrm{~nm}$ thick film.

For comparison, we show local thermal analysis measurements for ungrafted polystyrene (PS) films on $\mathrm{SiO}_{x}$ annealed at $130{ }^{\circ} \mathrm{C}$. As it lacks the grafting moiety - the $-\mathrm{OH}$ terminal groups - PS does not graft to $\mathrm{SiO}_{x}$. The $T_{g}$ 's of the PS films showed a trend opposite to that shown by the PS-OH films; the $T_{g}$ was reduced below the bulk value for films less than $60 \mathrm{~nm}$ thick. 


\section{DISCUSSION}

For films of PHS grafted to the silicon substrate we observe deviations from bulk behavior at a critical film thickness as high as $190 \mathrm{~nm}$. Previously, deviations from bulk behavior for ungrafted films had been observed only for films thinner than $60 \mathrm{~nm}$. Furthermore, large deviations from bulk $T_{g}$ had been observed only for ultrathin films: $70{ }^{\circ} \mathrm{C} T_{g}$ depression for $30 \mathrm{~nm}$ free-standing PS films, ${ }^{4}$ and $30^{\circ} \mathrm{C} T_{g}$ elevation for $20 \mathrm{~nm}$ PMMA films. ${ }^{29}$ We have shown that the $T_{g}$ of PHS can be elevated above the bulk value by $25^{\circ} \mathrm{C}$ for films as thick as $150 \mathrm{~nm}$, and by as much as $55^{\circ} \mathrm{C}$ for films $100 \mathrm{~nm}$ thick. As mentioned in the Introduction, the mechanical and transport properties of polymers in the bulk are related to their $T_{g}$ 's, thus the extraordinary elevations of $T_{g}$ obtainable by grafting may allow us to optimize or control the properties of nanoscale structures. Of course, the relationship between dimension dependent $T_{g}$ 's and mechanical and transport properties remains to be established for thin film geometries and other nanoscopic systems. Furthermore, $T_{g}$ is an isotropic property, whereas the mechanical or transport properties of films or nanoscopic systems may be anisotropic, in contrast to their behavior in the bulk.

For end-grafted PS-OH films, the critical thickness (110 $\mathrm{nm}$ ) is about double that observed for (ungrafted) PS films. However, while the increase in $h_{c}$ is dramatic for the endgrafted chains, it pales in comparison to the more than threefold increase in $h_{c}$ observed for the side-grafted PHS chains. Tsui et $a l^{46}{ }^{4}$ have also measured the $T_{g}$ 's of films on PS-OH brushes. They prepared their samples by: (1) depositing a PS-OH ( $\sim 10000 \mathrm{~g} / \mathrm{mol})$ brush ( $3 \mathrm{~nm}$ thick) by annealing a film of PS-OH on $\mathrm{SiO}_{x}$ at $170{ }^{\circ} \mathrm{C}$ for $48 \mathrm{~h}$, (2) removing the ungrafted PS-OH polymer by solvent extraction, and (3) spin coating a $33 \mathrm{~nm}$ film of PS (96000 g/mol) from toluene onto the brush covered substrate. Using x-ray reflectivity, they determined the $T_{g}$ of the PS film on the brush $\left(95^{\circ} \mathrm{C}\right)$ to be barely greater than the $T_{g}$ of a $33 \mathrm{~nm}$ PS film on native $\mathrm{SiO}_{x}$ $\left(92{ }^{\circ} \mathrm{C}\right)$. The thinnest film of grafted PS-OH we studied was $43 \mathrm{~nm}$ thick and had a $T_{g}$ that was $25^{\circ} \mathrm{C}$ above the bulk value. We attribute the differences between our results and those of Tsui et al. to: (1) differences in the thickness of the brush layer (19 nm vs $3 \mathrm{~nm}$ ), (2) differences in the samplepreparation methods and materials, and (3) differences in chain conformations especially at the interface between the grafted and ungrafted material. The difference between the $T_{g}$ measurements by Tsui et al. and ours and the apparent dependence of the glass transition temperature of thin polymer films on the "molecular architecture" of the film seems to be at variance with the results of Prucker et al. ${ }^{47}$ They reported that the glass transition temperatures of thin films of PMMA, measured using optical waveguide spectroscopy, were the same for three preparation techniques: spin casting, Langmuir-Blodgett-Kuhn deposition (multiple layer structure), and grafting-from (brush structure). The spin cast and LBK deposited films were prepared on HMDS treated hydrophobic surfaces and the $T_{g}$ depression observed is consistent with the results of Ref. 3. The lack of difference in $T_{g}(h)$ between grafted and ungrafted PMMA films calls for further study.

It is tempting to speculate that chain entanglement plays a role in the difference between our results and those of Tsui et al. and that it explains the moderately large critical thickness $(110 \mathrm{~nm})$ and $T_{g}$ elevations we have measured for endgrafted PS-OH films. The PS-OH in our samples is likely to be highly entangled since the $M_{W}$ we have used is $100 \mathrm{~kg} /$ mol, well above the molecular weight of entanglement of PS, $M_{e} \approx 17.7 \mathrm{~kg} / \mathrm{mol}^{48,49}$ For PHS, however, our data indicate that chain entanglement is unlikely to be an important factor in determining the thickness dependence of $T_{g}$ because: (1) the thickness of the grafted layer after a Soxhlet extraction in THF is within $10 \%$ of the thickness after the PGMEA wash, indicating that there is little entangled but ungrafted polymer in the residual layer, and (2) the molecular weights of the two samples of PHS we have studied are $10 \mathrm{~kg} / \mathrm{mol}$ and 20 $\mathrm{kg} / \mathrm{mol}$, below the $M_{e}$ of PHS. ${ }^{50,51}$

In terms of our previous work demonstrating the effect of interfacial energy on the $T_{g}$ of supported films, chains grafted to the substrate could be viewed as constituting a very high-energy interface. For nongrafted interfaces, $T_{g}$ increased linearly with increasing interfacial energy at constant film thickness. Information about the molecular architecture of the film is required to establish a quantitative connection between grafting and interfacial energy. The surface and interfacial energy can be determined by integrating the difference between the tangential and normal components of the stress tensor in the interfacial region..$^{52}$ The stress tensor depends on the interactions between the polymer and itself and the polymer and the substrate or air interface, respectively. These interactions lead to different chain configurations and packing arrangements, which in turn result in specific values of the surface and interfacial energy. In the case of grafted chains or polymer brushes, the interpenetration of the polymer and the "bound" layer or the brush, are likely to have important effects on the local stresses, thereby having pronounced effects on the interfacial energy. We are currently examining these issues by means of molecular simulations, and the results of this work will be presented in a future publication.

The large critical thicknesses we have observed are puzzling. On the one hand, discussions of previous results in the literature claimed that chain dimensions are not necessarily a relevant parameter when considering length scales determining the $T_{g}$ of thin films. ${ }^{1}$ Except for the data on unsupported PS films of very high $M_{W}\left(\approx 575 \times 10^{3}-9.1 \times 10^{6} \mathrm{~g} / \mathrm{mol}\right)$ reported in Refs. 8 and 53, no molecular weight (and hence, chain dimension) dependence of $T_{g}(h)$ has been reported. On the other hand, molecular dynamics simulations, ${ }^{54}$ for example, reveal that: (1) increased or decreased segmental mobility persists into the film for distances $\geqslant 10 R_{g}$ from a free surface and an attractive substrate respectively and (2) $h_{c}=15 R_{g}$ for supported films on substrates with interaction potentials that lead to $T_{g}$ depression and $T_{g}$ elevation. Equally puzzling is the fact that most of our $T_{g}$ results were obtained with local thermal analysis. An analytical solution of the thermal probe heat flow model in Ref. 3 shows that the temperature beneath the probe varies approximately linearly with depth between its value at the probe tip and its value at the substrate. Only the outermost 5\% of the film (approx. 10 $\mathrm{nm}$ or less) is within $5 \%$ of the probe temperature. Local 
thermal analysis seems to be sensitive primarily to surface layer properties. In the case of PHS, H-bonding may be a possible explanation for the persistence of near-interface behavior well into the film and the large critical thicknesses obtained, but by itself fails to explain the dependence of $T_{g}$ on film thickness.

There have been a number of attempts to understand the thickness dependence of the relative $T_{g}$ of thin polymer films. ${ }^{1,5,8,10,31,32,53-58}$ Most of the analyses are only applicable to $T_{g}$ reductions; if they mention $T_{g}$ elevations at all, many of the authors of the aforementioned references themselves caution against extending their analysis to $T_{g}$ elevations. Previous reports of experiments demonstrating $T_{g}$ elevation $^{3,59}$ have not attempted any empirical fit of their $T_{g}(h)$ data, though the authors do attribute it to the attractive polymer-substrate interfacial interaction energy, and later experiments have demonstrated this relationship quantitatively. ${ }^{29}$

Forrest et al. ${ }^{8}$ used a piecewise linear form to describe the $T_{g}(h)$ depression observed for free-standing PS films of $M_{W} \geqslant 575 \mathrm{~kg} / \mathrm{mol}$. In an argument set forth by de Gennes ${ }^{31}$ and Forrest et al. ${ }^{32}$ the abrupt transition at the critical thickness between bulklike and dimension dependent behavior that is shown by the data indicates the existence of two competing mechanisms for the increase in mobility with temperature: the bulk mechanism that dominates for thick films and a distinct mechanism that dominates at sub-critical film thickness. Our data showing elevation in $T_{g}$ with decreasing film thickness are also best fit with a piecewise linear form [represented by Eq. (1)] in which an abrupt transition is implicit, as we have seen in Sec. III B. de Gennes and Forrest et al. proposed the dominance of a higher mobility mode in films of sub-critical thickness to explain the linear $T_{g}$ depressions that the films exhibit. Clearly, this explanation is not applicable to describe $T_{g}$ elevations. For $T_{g}$ elevations relative to bulk, the dominant mode for mobility change with temperature in that regime is a lower mobility mode. The only manner in which a lower mobility mode can dominate a higher mobility mode is if the higher mobility mode actually turns off.

Although the piecewise linear function provides a good fit to our data, another model is commonly used to describe the $T g(h)$ behavior of thin polymer films. Keddie et al. ${ }^{1}$ use the concepts of cooperative motion and postulate a temperature dependent length scale $\xi$ associated with a liquidlike surface layer [ibid, Eq. (2)]:

$$
\xi(T)=\xi_{0}\left(1-\frac{T}{T_{g}^{\text {bulk }}}\right)^{-1 / \delta}, \quad\left(T<T_{g}^{\text {bulk }}\right),
$$

where $\xi_{0}$ is the length scale at low temperatures and $\delta$ is a positive exponent in order to ensure that $\xi$ is singular as $T$ $\rightarrow T_{g}^{\text {bulk }}$. Based on the above, they justify fitting their data to the empirical relation [ibid, Eq. (1)]

$$
T_{g}(h)=T_{g}^{\text {bulk }}\left(1-\left(\frac{\xi_{0}}{h}\right)^{\delta}\right) .
$$

Using the same value of the exponent as Keddie et al., ${ }^{1} \delta$ $=1.8$, for our ungrafted PS data (see Fig. 3) we obtain a best fit value of $\xi_{0}=3.3 \pm 0.2 \mathrm{~nm}$, in good agreement with their value of $3.2 \mathrm{~nm}$. Equation (3), however, is obviously inappropriate to describe $T_{g}$ elevations. ${ }^{60}$

We have attempted to find an empirical form for our data based on the above mobility length scale argument. Note that there is a duality between an unconstrained surface layer of high mobility and a grafted layer of low mobility. If we let $\beta_{g}=1 / T_{g}$ be the inverse glass transition temperature, we see the duality as follows:

Surface layer $\leftrightarrow$ Grafted layer

$$
\left(T_{g}^{\text {surface }} \rightarrow 0\right) \leftrightarrow\left(\beta_{g}^{\text {grafted }} \rightarrow 0 \text {, i.e., } T_{g}^{\text {grafted }} \rightarrow \infty\right)
$$

$T_{g}$ reductions $\leftrightarrow \beta_{g}$ reductions, i.e., $T_{g}$ elevations.

This duality suggests that we replace $T_{g}$ with $\beta_{g}$ in Eq. (3) above and leads us to postulate the following empirical dependence of the glass transition temperature on the film thickness:

$$
\beta_{g}(h)=\beta_{g}^{\text {bulk }}\left(1-\left(\frac{\xi_{0}}{h}\right)^{\delta}\right) .
$$

In terms of length scales for mobility

$$
\xi(T)=\xi_{0}\left(1-\frac{T_{g}^{\text {bulk }}}{T}\right)^{-1 / \delta}, \quad\left(T>T_{g}^{\text {bulk }}\right)
$$

one has the following interpretation: $\xi$ is a length scale for mobility reduction, as suggested by the above duality, and is singular as $T \rightarrow T_{g,+}^{\text {bulk }}$ (i.e., from above) and $\stackrel{\substack{T \rightarrow \infty \\ \rightarrow}}{\rightarrow} \xi_{0}$, which one can interpret as the thickness of the immobile layer.

Unfortunately, Eq. (4) does not yield consistently good fits to our sets of data showing $T g$ elevations, since, in general, either the estimated variance of the data from the fit or the estimated errors in the fitting parameters are very large. This brings into question the validity of models of dimension dependent $T_{g}$ that are based on distinct reduced mobility layers. Any multi-layer model would in fact lead to a smooth $T_{g}(h)$. Forrest $e t a l .{ }^{8}$ also argue that the piecewise and nonsmooth nature of $T_{g}(h)$ contradicts a model based on the existence of a liquidlike surface (enhanced mobility) layer. Experimental indications of the existence of a liquid layer, however, continue to be reported; ${ }^{10}$ and evidence for a liquid layer model is provided by Wallace et al. ${ }^{61}$ who have shown that chain relaxation at the surface occurs $50 \%$ faster at the surface than in the bulk.

For the data pertaining to PHS20 films annealed at $160{ }^{\circ} \mathrm{C}$ the above model based on the reduced mobility layer provides a better fit [Eq. (4), with the scale $\xi_{0} \approx 58 \pm 5 \mathrm{~nm}$ and the exponent $\delta \approx 4.9 \pm 0.6]$ than does the dual mechanism model, Eq. (1). Interestingly, these data were obtained by means of ellipsometry. Although, as we have emphasized before, $T_{g}$ values obtained by the use of ellipsometry and local thermal analysis are in very good agreement with each other, the two techniques may differ in their sensitivity to the onset of a nonbulklike dominant mechanism as the film thickness is reduced below its critical value, accounting for the apparent difference in the sharpness of this transition. 


\section{CONCLUSION}

Grafting polymer chains (or portions of chains) at the interface between supported thin polymer films and the substrate leads to substantial increases both in the critical thickness below which the $T_{g}$ of the film deviates from that of the bulk polymer and in the degree of elevation of $T_{g}$ from the bulk value with decreasing film thickness. For films in which polymer chains are not grafted to the substrate, the $T_{g}$ 's of supported polymer films have been observed to increase or decrease relative to the bulk polymer depending on the type of substrate and the interfacial energy of the substrate, but only for critical film thicknesses less than $50 \mathrm{~nm}$. By endgrafting PS chains near the substrate, the critical thickness for an elevation in $T_{g}$ relative to the bulk was $110 \mathrm{~nm}$, double the critical thickness for ungrafted films, resulting in a $T_{g}$ elevation of $25^{\circ} \mathrm{C}$ for a $43 \mathrm{~nm}$ thick film. By sidegrafting PHS chains to the substrate by annealing films at $190{ }^{\circ} \mathrm{C}$, the critical thickness was almost $200 \mathrm{~nm}$, resulting in a $T_{g}$ elevation of more than $40^{\circ} \mathrm{C}$ for a $100 \mathrm{~nm}$ thick film. Dimension dependent material properties are anticipated to play an important role in the microelectronics industry in the next few years. The relevant dimension of polymer structures in the lithographic process is currently $100 \mathrm{~nm}$; resist thickness for advanced exposure systems may approach this dimension, and the in-plane dimensions of post-patterning resist structures will certainly be of this scale and smaller. ${ }^{62}$ In the present work we have demonstrated that polymer structures with just one small dimension (films) of $100 \mathrm{~nm}$ have $T_{g}$ 's that deviate significantly from that of the bulk polymer. It remains to be established that properties of amorphous polymers related to $T_{g}$, i.e., transport and mechanical properties, exhibit the same dimensional dependence. In addition, there is little or no information about the dimension dependent properties of nanoscopic structures that have two or three dimensions of sub-100 nm scale. In spite of this, the phenomena we have described will have to be taken into account during the processing of resist materials in advanced lithography, and may in fact offer opportunities for designing resist systems with improved properties and performance at the sub-100 nm scale.

\section{ACKNOWLEDGMENTS}

The authors thank Qijang Gu for help with synthesizing the hydroxyl terminated polystyrene. Two of the authors (R.S.T.) and (S.P.) would like to thank A. Pawloski, R. Peters, and N. Ekdawi for help with various instruments, and I. Echeverría, M. Ediger, T.S. Jain, and Hyuk Yu for discussions. Funding for this work was provided by the Semiconductor Research Corporation (Grant No. 98-LP-452), NSF Career Award (Grant No. CTS-9703207), and the Camille Dreyfus Teacher-Scholar Awards Program. Facilities were supported by DARPA/ONR (Grant No. N00014-97-1-0460) and the NSF (Grant No. DMR-0084402). The Synchotron Radiation Center at the University of Wisconsin-Madison is supported by the NSF under Award No. DMR-9531009.
${ }^{1}$ J. L. Keddie, R. A. L. Jones, and R. A. Cory, Europhys. Lett. 27, 59 (1994).

${ }^{2}$ W. E. Wallace, J. H. van Zanten, and W. L. Wu, Phys. Rev. E 52, R3329 (1995).

${ }^{3}$ D. S. Fryer, P. F. Nealey, and J. J. de Pablo, Macromolecules 33, 6439 (2000).

${ }^{4}$ J. A. Forrest, K. Dalnoki-Veress, J. R. Stevens, and J. R. Dutcher, Phys. Rev. Lett. 77, 2002 (1996).

${ }^{5}$ J. A. Forrest, K. Dalnoki-Veress, and J. R. Dutcher, Phys. Rev. E 56, 5705 (1997)

${ }^{6}$ N. Satomi, A. Takahara, and T. Kajiyama, Macromolecules 32, 4474 (1999)

${ }^{7}$ G. B. DeMaggio, W. E. Frieze, D. W. Gidley, M. Zhu, H. A., Hristov, and A. F. Yee, Phys. Rev. Lett. 78, 1524 (1997).

${ }^{8}$ J. A. Forrest and K. Dalnoki-Veress, Adv. Colloid Interface Sci. (in press).

${ }^{9}$ J. A. Forrest and R. A. L. Jones, in Polymer Surfaces, Interfaces and Thin Films, edited by A. Karim and S. Kumar (World Scientific, Singapore, 2000).

${ }^{10}$ S. Kawana and R. A. L. Jones, Phys. Rev. E 63, 021501 (2001).

${ }^{11}$ J. L. Keddie, R. A. L. Jones, and R. A. Cory, Faraday Discuss. 98, 219 (1994).

${ }^{12}$ O. Kahle, U. Wielsch, H. Metzner, J. Bauer, C. Uhlig, and C. Zawatzki, Thin Solid Films 313, 803 (1998).

${ }^{13}$ Y. Grohens, M. Brogly, C. Labbe, M. O. David, and J. Schultz, Langmuir 14, 11 (1998).

${ }^{14}$ J. H. Kim, J. Jang, and W. C. Zin, Langmuir 16, 4064 (2000).

${ }^{15}$ J. H. van Zanten, W. E. Wallace, and W. L. Wu, Phys. Rev. E 53, R2053 (1996).

${ }^{16}$ C. W. Frank, V. Rao, M. M. Despotopoulou, R. F. W. Pease, W. D. Hinsberg, R. D. Miller, and J. F. Rabolt, Science 273, 912 (1996).

${ }^{17}$ D. B. Hall and L. M. Torkelson, Macromolecules 31, 8817 (1998).

${ }^{18}$ B. Frank, A. P. Gast, T. P. Russell, H. R. Brown, and C. J. Hawker, Macromolecules 29, 6531 (1996).

${ }^{19}$ X. Zheng, M. H. Rafailovich, J. Sokolov, Y. Strzhemechny, S. A. Schwarz, B. B. Sauer, and M. Rubinstein, Phys. Rev. Lett. 79, 241 (1997).

${ }^{20}$ E. K. Lin, W. L. Wu, and S. K. Satija, Macromolecules 30, 7224 (1997).

${ }^{21}$ J. A. Forrest, K. Dalnoki-Veress, and J. R. Dutcher, Phys. Rev. E 58, 6109 (1998).

${ }^{22}$ Y.-C. Lee, K. C. Bretz, F. W. Wise, and W. H. Sachse, Appl. Phys. Lett. 69, 1692 (1996).

${ }^{23}$ K. C. Bretz, Y.-C. Lee, F. W. Wise, and W. H. Sachse, Bull. Am. Phys. Soc. 42, 648 (1997).

${ }^{24}$ K. van Workum, P. F. Nealey, and J. J. de Pablo (unpublished).

${ }^{25}$ H. H. Solak, D. He, W. Li, and F. Cerrina, J. Vac. Sci. Technol. B 17, 3052 (1999).

${ }^{26}$ H. B. Cao, P. F. Nealey, and W.-D. Domke, J. Vac. Sci. Technol. B 18, 3303 (2000)

${ }^{27}$ W.-M. Cheng, G. A. Miller, J. A. Manson, R. W. Hertzberg, and L. H. Sperling, J. Mater. Sci. 25, 1917 (1990).

${ }^{28}$ D. W. Van Krevelen, Properties of Polymers (Elsevier, Amsterdam, 1990), p. 383.

${ }^{29}$ D. S. Fryer, R. D. Peters, Eui Jun Kim, J. E. Tomaszewski, J. J. de Pablo, P. F. Nealey, C. C. White, and Wen-li Wu, Macromolecules 34, 5627 (2001).

${ }^{30}$ S. T. Milner, Science 251, 905 (1991).

${ }^{31}$ P. G. de Gennes, Eur. Phys. J. E 2, 210 (2000)

${ }^{32}$ K. Dalnoki-Veress, J. A. Forrest, P. G. de Gennes, and J. R. Dutcher, J. Phys. IV 10, 221 (2000).

${ }^{33}$ D. J. Gravert and K. D. Janda, Tetrahedron Lett. 39, 1513 (1998).

${ }^{34}$ S. Srinivasan, R. Twieg, J. L. Hedrick, and C. J. Hawker, Macromolecules 29, 8543 (1996).

${ }^{35}$ A. Hammiche, M. Reading, H. M. Pollock, M. Song, and D. J. Hourston, Rev. Sci. Instrum. 67, 4268 (1996).

${ }^{36}$ A. Hammiche, L. Bozec, M. Conroy, H. M. Pollock, G. Mills, J. M. R. Weaver, D. M. Price, M. Reading, D. J. Hourston, and M. Song, J. Vac. Sci. Technol. B 18, 1322 (2000).

${ }^{37}$ R. J. Pylkki, P. J. Moyer, and P. E. West, Jpn. J. Appl. Phys., Part 1 33, 3785 (1994)

${ }^{38}$ J. R. Dutcher, J. A. Forrest, and K. Dalnoki-Veress, Abstr. Pap. - Am. Chem. Soc. 215, 276 (1998).

${ }^{39}$ R. A. L. Jones, R. J. Lehnert, H. Schönherr, and J. Vansco, Polymer 40, 525 (1999).

${ }^{40}$ A. Sidorenko, S. Minko, K. Schenk-Meuser, H. Duschner, and M. Stamm, Langmuir 15, 8349 (1999). 
${ }^{41}$ S. Arichi, N. Sakamoto, and M. Yoshida, Polymer 27, 1761 (1986).

${ }^{42}$ R. Jordan, A. Ulman, J. F. Kang, M. A. Rafailovich, and J. Sokolov, J. Am. Chem. Soc. 121, 1016 (1999).

${ }^{43}$ J. Brandrup and E. H. Immergut, Polymer Handbook, 3rd ed. (Wiley Interscience, New York, 1988), p. VII/38.

${ }^{44}$ Y. Miyaki, Y. Elinaga, and H. Fujita, Macromolecules 11, 1180 (1978).

${ }^{45}$ P. Mansky, Y. Liu, E. Huang, T. P. Fussell, and C. Hawker, Science 275, 1458 (1997).

${ }^{46}$ O. K. C. Tsui, T. P. Russell, and C. J. Hawker, J. Am. Chem. Soc. (in press).

${ }^{47}$ O. Prucker, S. Christian, H. Bock, J. Rühe, C. W. Frank, and W. Knoll, Macromol. Chem. Phys. 199, 1435 (1998).

${ }^{48}$ S. Onogi, T. Moruga, and K. Kitagawa, Macromolecules 3, 109 (1970).

${ }^{49}$ D. J. Plazek, A. Riande, H. Markowitz, and N. Ragupathi, J. Polym. Sci., Part B: Polym. Phys. 17, 2189 (1979).

${ }^{50}$ L. J. Fetters, D. J. Lohse, and W. W. Graessley, J. Polym. Sci., Part B: Polym. Phys. 37, 1023 (1999).

${ }^{51}$ L. J. Fetters, D. J. Lohse, and S. T. Milner, Macromolecules 32, 6847 (1999).
${ }^{52}$ F. Vernik, J. Baschnagel, and K. Binder, J. Chem. Phys. 113, 4444 (2000).

${ }^{53}$ K. Dalnoki-Veress, J. A. Forrest, C. Murray, C. Gigault, and J. R. Dutcher, Phys. Rev. E 63, 031801 (2001).

${ }^{54}$ J. A. Torres, P. F. Nealey, and J. J. de Pablo, Phys. Rev. Lett. 85, 3221 (2000).

${ }^{55}$ Q. Jiang, H. X. Shi, and J. C. Li, Thin Solid Films 354, 283 (1999).

${ }^{56}$ J. A. Forrest and J. Mattson, Phys. Rev. E 61, R53 (2000).

${ }^{57}$ K. L. Ngai, Eur. Phys. J. E (special issue) Properties of Thin Polymer Films (submitted).

${ }^{58}$ S. Herminghaus, K. Jacobs, and R. Seeman, "The glass transition of thin polymer films: some questions and a possible answer," University of Ulm preprint (2001).

${ }^{59}$ J. L. Keddie, R. A. L. Jones, and R. A. Cory, Faraday Discuss. 98, 219 (1994).

${ }^{60}$ The function obtained by changing the sign of the second term in parentheses does work.

${ }^{61}$ W. E. Wallace, D. A. Fischer, K. Efimenko, Wen-Li Wu, and J. Genzer, Macromolecules 34, 5081 (2001).

${ }^{62}$ Y. Vladimirsky, Proc. SPIE 3676, Part 1, xvi (1999). 